\title{
Stability analysis of the car-trailer system with a time-delayed tyre model
}

\author{
S. Beregi, D. Takács, G. Stépán \\ MTA-BME Research Group on Dynamics of Machines and Vehicles, Department of Applied Mechanics, \\ Budapest University of Technology and Economics, Budapest, Hungary \\ D.A.W. Barton \\ Department of Engineering Mathematics, Bristol, United Kingdom
}

\begin{abstract}
In this paper we investigate the dynamics of towed vehicles by means of two examples, the car-trailer combination and a towed trailer, both represented by the so-called single track model. The effect of tyre deformation is considered with the help of the brush tyre model. In our analysis we take into account the so-called 'memory effect' in the tyre-ground contact patch too, which leads to delay differential equations with distributed time-delay as governing equations. The stability of the rectilinear motion is analysed by means of stability charts with respect to the vehicle parameters. For the towed trailer we also performed nonlinear analysis which enabled us to capture the Hopf bifurcations emerging at the stability boundaries. These were compared with numerical simulation, where we studied the effect of lateral tyre-slip, too.
\end{abstract}

Keywords: tyre/road interaction, car-trailer lateral-stability, memory effect in tyres, self-excited vibrations, nonlinear analysis

\section{INTRODUCTION}

The deformation of rubber tyres have a significant role in the dynamics of road vehicles as the forces and aligning torques generated in the small contact regions strongly influence the large scale motion of the vehicles. Therefore, understanding the behaviour of the tyres is essential for developing vehicle-models, which explains the fact that tyre dynamics is one of the core fields of research in vehicle dynamics.

Several tyre models have been developed to be used in analytical and numerical investigations of vehicle systems. The simplest models assume quasi-static tyre deformation (Pacejka, 2002) both inside and outside the tyre-ground contact patch. This approach became a standard in vehicle dynamics due to the fact that these models can efficiently be used for different vehicle set-ups running with large or medium velocities (e.g. aircraft ground dynamics (Coetzee et al., 2006), (Terkovics et al., 2012), (Howcroft et al., 2012) or the analysis of the cornering manoeuvres of automobiles (Rossa et al., 2012)). However, in the case when the instantaneous shape of the deformed tyre is relevant more accurate models are needed.

In this paper we study the stability of towed vehicles with the help of the so-called single-track model (Pacejka, 2002). In terms of tyre modelling we focus on the accurate calculation of the tyre deformation in the contact patch by means of a time-delayed tyre model, which can capture the so-called regenerative vibrations of the tyres (Takács and Stépán, 2012).

In order to understand the nature of the emerging self-excited vibrations, we also perform nonlinear analysis. As these calculations are computationally expensive, to keep our equations manageable, we concentrate on the dynamics of the trailer instead of the full car-trailer combination. Firstly, we focus on the linear stability analysis, which provides us the stability boundaries belonging to Hopf bifurcations, which occur with a similar structure as for the car-trailer system. To calculate the corresponding periodic orbits of the governing equations the method of collocation is used (Trefethen, 2000). 


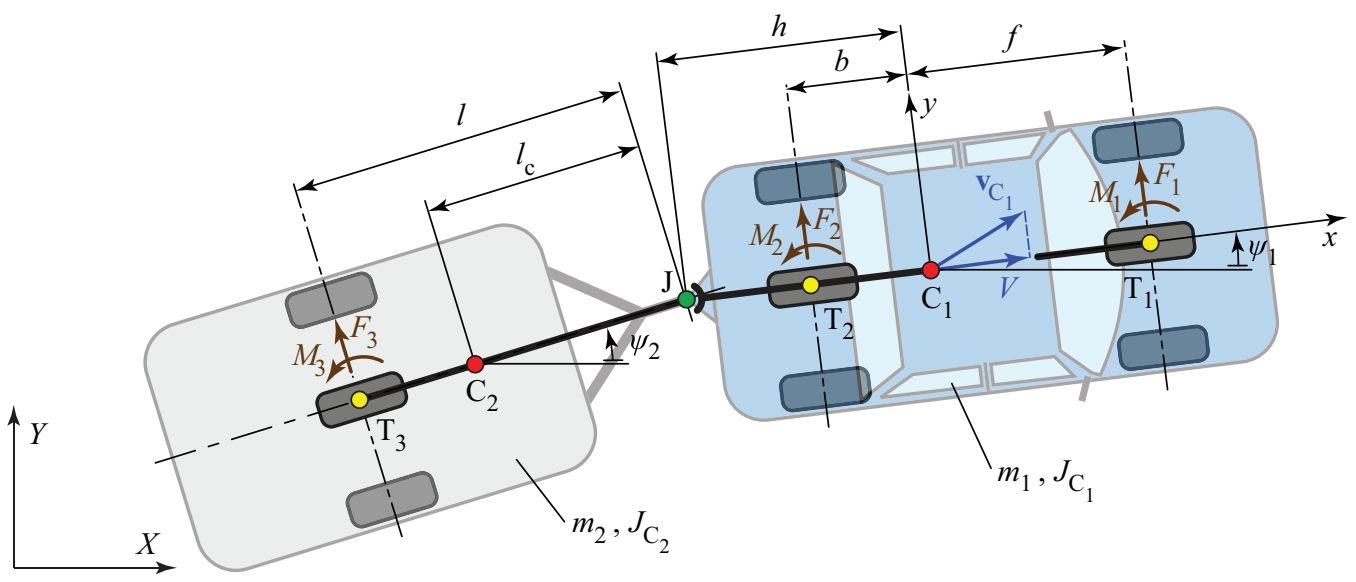

Figure 1. The single-track model of the car-trailer combination

Finally, to investigate the impact of the (possible) partial slip of the tyres we enhance our tyre model by taking into account the effect of friction between the tyre and the road surface, which leads to a piecewise-smooth system. Although the calculation of periodic orbits gets computationally expensive in this case, we can still get an insight of the behaviour of the system by performing numerical simulations which may determine the direction of further studies as well.

\section{MECHANICAL MODEL}

\subsection{The car-trailer combination}

In our study we investigate the motion of the vehicles in the $(X, Y)$ plane. As shown in Figure 1 the car and the trailer are modelled by the so-called single-track model, which is often also referred as the 'bicycle model' in the specific literature (Pacejka, 2002). Thus, the car-trailer system is represented by two rods connected with a rotational joint at point $\mathrm{J}$.

The tyres are mounted to the vehicle at points $T_{1}, T_{2}$ and $T_{3}$. It is assumed that each tyre is acting on the vehicle-system with a force $F_{i}$ and an aligning torque $M_{i}(i=1,2,3)$. The distances of the front and rear tyres from the centre of gravity $\mathrm{C}_{1}$ of the towing car are denoted by $f$ and $b$ respectively, whereas the distance between the centre of gravity $\mathrm{C}_{1}$ and the rotational joint $\mathrm{J}$ is $h$. The distance of the centre of gravity $\mathrm{C}_{2}$ of the trailer from the king pin $\mathrm{J}$ is referred to $l_{\mathrm{c}}$, while the parameter $l$ (often called caster/trail length) describes the distance between the king pin $\mathrm{J}$ and the wheel axle of the trailer. The model has four degrees of freedom (DoF), hence we choose four generalized coordinates to describe the motion of the vehicle: $X_{1}(t)$ and $Y_{1}(t)$, which are the $X$ and $Y$ coordinates of the centre of gravity $\mathrm{C}_{1}$, and the deflection angles $\psi_{1}(t)$ and $\psi_{2}(t)$ of the longitudinal axes of the car and the trailer with respect to the $X$ direction.

Moreover, a kinematic constraint is applied to the system, namely, the longitudinal velocity $V$ of the car centre of gravity is kept constant in time:

$$
\dot{X}_{1}(t) \cos \psi_{1}(t)+\dot{Y}_{1}(t) \sin \psi_{1}(t)=V,
$$

where the dots refer to the derivatives with respect to time. The presence of the kinematic constraint provides a non-holonomic mechanical system, the equation of motion of which can be efficiently determined by means of the Appell-Gibbs equation (Gantmacher, 1975). This requires the introduction of the so-called pseudo velocities $\beta_{r}(t) r=1,2,3$ :

$$
\beta_{1}(t)=-\dot{X}_{1}(t) \sin \psi_{1}(t)+\dot{Y}_{1}(t) \cos \psi_{1}(t), \quad \beta_{2}(t)=\dot{\psi}_{1}(t), \quad \beta_{3}(t)=\dot{\psi}_{1}(t) .
$$

where $\beta_{1}(t)$ is the lateral velocity of centre of gravity $\mathrm{C}_{1}$ of the car, while $\beta_{2}(t)$ and $\beta_{3}(t)$ are the angular velocities of the car and the trailer, respectively. 
With the help of these the following equations of motion can be derived:

$$
\begin{aligned}
& m_{1}\left(\dot{\beta}_{1}(t)+V \beta_{2}(t)\right)+m_{2}\left(\dot{\beta}_{1}(t)+V \beta_{2}(t)-h \dot{\beta}_{2}(t)\right. \\
& \quad-l_{\mathrm{C}} \dot{\beta}_{3}(t) \cos \left(\psi_{2}(t)-\psi_{1}(t)\right)+l_{\mathrm{C}} \beta_{3}^{2}(t) \sin \left(\psi_{2}(t)-\psi_{1}(t)\right) \\
& \quad=F_{1}+F_{2}+F_{3} \cos \left(\psi_{2}(t)-\psi_{1}(t)\right) \\
& J_{\mathrm{C}_{1}} \dot{\beta}_{2}(t)+m_{2}\left(h^{2} \dot{\beta}_{2}(t)-h V \beta_{2}(t)-h \dot{\beta}_{1}(t)\right. \\
& \quad+h l_{\mathrm{C}} \dot{\beta}_{3}(t) \cos \left(\psi_{2}(t)-\psi_{1}(t)\right)-h l_{\mathrm{C}} \beta_{3}^{2} \sin \left(\psi_{2}(t)-\psi_{1}(t)\right) \\
& \quad=F_{1} f-F_{2} b-F_{3} h \cos \left(\psi_{2}(t)-\psi_{1}(t)\right)+M_{1}+M_{2}, \\
& J_{\mathrm{C}_{1}} \dot{\beta}_{2}(t)+m_{2}\left(l_{\mathrm{C}}^{2} \dot{\beta}_{3}(t)-l_{\mathrm{C}} V \beta_{2}(t) \cos \left(\psi_{2}(t)-\psi_{1}(t)\right)-l_{\mathrm{C}} \dot{\beta}_{1}(t) \cos \left(\psi_{2}(t)-\psi_{1}(t)\right)\right. \\
& \quad+l_{\mathrm{C}} \beta_{1}(t) \beta_{2}(t) \sin \left(\psi_{2}(t)-\psi_{1}(t)\right)+h l_{\mathrm{C}} \dot{\beta}_{2}(t) \cos \left(\psi_{2}(t)-\psi_{1}(t)\right) \\
& \quad+h l_{\mathrm{C}} \beta_{2}^{2}(t) \sin \left(\psi_{2}(t)-\psi_{1}(t)\right)=M_{3}-F_{3} l,
\end{aligned}
$$

where $m_{1}$ and $m_{2}$ are the masses, $J_{\mathrm{C}_{1}}$ and $J_{\mathrm{C}_{2}}$ are the mass moments of inertia about the $Z$ axis at the centres of gravity of the car and the trailer, respectively.

\subsection{Towed trailer}

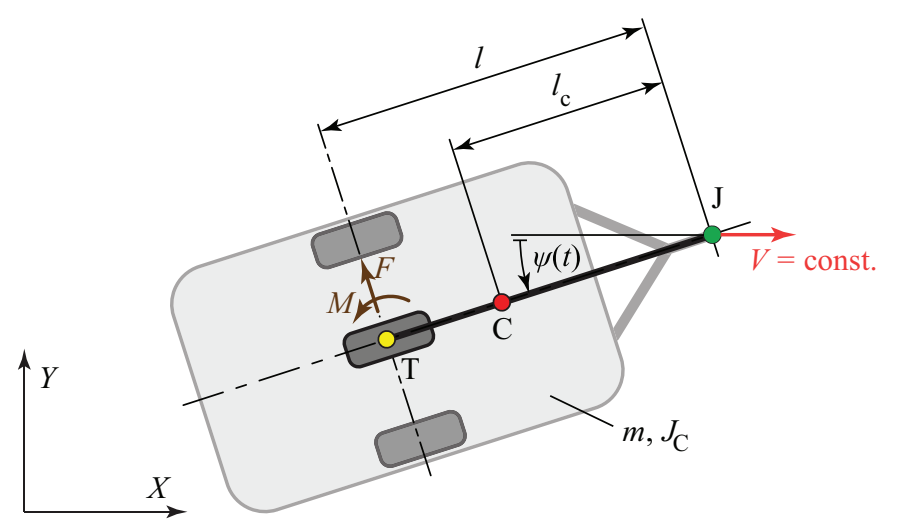

Figure 2. The single-track model of the trailer towed along the $X$ direction with a constant velocity $V$.

As it involves complex calculations, the nonlinear analysis is performed on a reduced system, where we only consider a trailer towed at the king pin $\mathrm{J}$ along the $X$-direction with a constant speed $V$, as shown in Figure 2.

This system has $1 \mathrm{DoF}$ as the orientation of the trailer in the $(X, Y)$ coordinate-plane is described by the deflection angle $\psi(t)$ only. Similarly to the car-trailer system, the distance between the centre of gravity $\mathrm{C}$ and the king pin $\mathrm{J}$ is denoted by $l_{\mathrm{C}}$, while the caster length $l$ describes the distance between the king pin $\mathrm{J}$ and the wheel axle of the trailer.

Using the constant towing speed $V$ one can prescribe a time-dependent geometric constraint for the king pin $\mathrm{J}$ as

$$
\mathbf{R}_{\mathbf{J}}(t)=\left(\begin{array}{lll}
V t & 0 & 0
\end{array}\right)^{T} .
$$

By means of these assumptions we can use the Lagrange equation of the second kind to provide the equation of motion, which can be expressed as

$$
\left(J_{\mathrm{C}}+m l_{\mathrm{C}}^{2}\right) \ddot{\psi}(t)=-F l+M,
$$

where $m$ is the mass of the trailer and $J_{\mathrm{C}}$ is its mass moment of inertia with respect to its centre of gravity $\mathrm{C}$. The concentrated force and the aligning torque originated from tyre deformation are denoted by $F$ and $M$, respectively.

\subsection{Tyre model}

In our studies we use the in-plane brush tyre model (Pacejka, 2002), (Takács, 2010) (see Fig. 3), by which we assume, that the tread and the carcass is modelled by infinitesimal massless particles with a linearly distributed lateral stiffness $k$. Thus, we assume, that the tyre deformation depends 


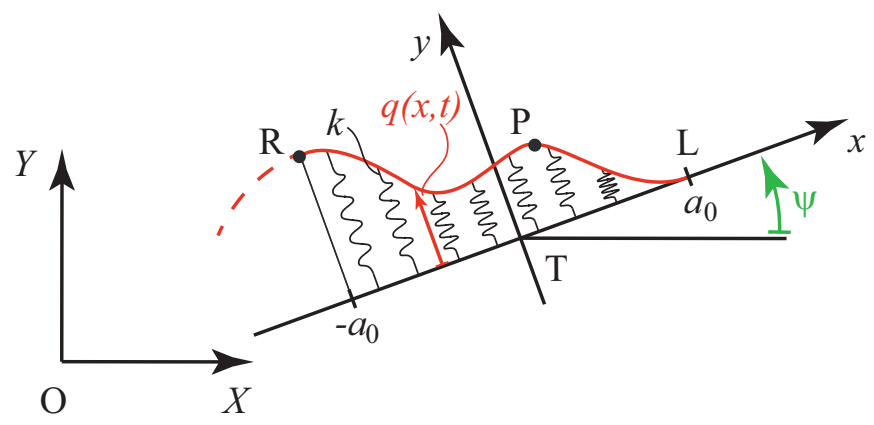

Figure 3. The in plane brush tyre model using the exact tyre deformation

on the local lateral load only, which means that outside the $x \in[-a, a]$ contact patch, where the lateral load is assumed to be zero, no deformation is considered.

To describe tyre deformation we consider two coordinate-systems as it is shown in Figure 3. Namely, the Lagrangian (or reference) system $(X, Y)$ with the origin $\mathrm{O}$, and the Eulerian system $(x, y)$ with the origin $\mathrm{T}$ moving together with the centre of the tyre-rim.

In case of small deformations it is an acceptable approximation to assume pure rolling in the contact patch, which can be formulated as a kinematic constraint: $\mathrm{d}_{\mathrm{P}} / \mathrm{d} t=0$ for $x \in[-a, a]$, where $\mathbf{R}_{\mathrm{P}}$ is the position vector of an arbitrary point $\mathrm{P}$ of the contact patch given in the Lagrangian system.

Using this, a nonlinear partial differential equation (PDE) can be derived which describes the exact deformation in the contact patch for pure rolling:

$$
\begin{aligned}
& \dot{q}(x, t)=\dot{X}_{\mathrm{T}}(t) \sin \psi(t)-\dot{Y}_{\mathrm{T}}(t) \cos \psi(t)-x \dot{\psi}(t) \\
& \quad+q^{\prime}(x, t)\left(\dot{X}_{\mathrm{T}}(t) \cos \psi(t)+\dot{Y}_{\mathrm{T}}(t) \sin \psi(t)-\dot{\psi}(t) q(x, t)\right)
\end{aligned}
$$

for $x \in[-a, a]$, where $\psi(t)$ denotes the deflection angle of the longitudinal axis $x$ of the tyre rim and the axis $X$. According to the implemented brush tyre model, the deformation is zero at the leading edge $\mathrm{L}$, which adds the boundary condition of $q(a, t)=0$ to the PDE above. Thus, the force $F$ and the aligning torque $M$ caused by tyre deformation can be calculated by integral formulae and the governing equations form a system of three integro-differential equations (IDEs) and three PDEs. For the purpose of our studies we transform them into a more manageable form. Namely, we can compose the travelling wave solution of the PDE; thus, we obtain a formula for the deformation with distributed time delay, which still represents the infinite dimensional behaviour of the system.

\subsection{The travelling wave solution}

In order to compose the travelling wave solution of the PDE in Eq. (6), we use the position vector $\mathbf{R}_{\mathrm{P}}$ of an arbitrary contact point (given in the reference coordinate system). Using this the travelling wave solution can be composed as

$$
\mathbf{R}_{\mathrm{P}}(x, t)=\mathbf{R}_{\mathrm{P}}(a, t-\tau(x)),
$$

for $x \in[-a, a]$ where $\tau(x)$ is the time delay, which is dependent to the spatial coordinate $x$. Based on this we can derive the solution for the lateral deformation $q(x(\tau), t)$ which satisfies the PDE Eq. (6) with a boundary condition of $q(a, t)=0$ :

$$
\begin{aligned}
& q(x(\tau), t)=\left(X_{T}(t)-X_{T}(t-\tau)\right) \sin \psi(t)-\left(Y_{T}(t)-Y_{T}(t-\tau)\right) \cos \psi(t) \\
& -a \sin (\psi(t)-\psi(t-\tau)) .
\end{aligned}
$$

Additionally, we obtain an expression, which describes coordinate $x$ :

$$
\begin{aligned}
x(\tau) & =-\left(X_{T}(t)-X_{T}(t-\tau)\right) \cos \psi(t)+\left(Y_{T}(t)-Y_{T}(t-\tau)\right) \sin \psi(t) \\
& +a \cos (\psi(t)-\psi(t-\tau)) .
\end{aligned}
$$


With the help of the formulae above, the tyre forces and aligning torques can be calculated as:

$$
F=-k \int_{0}^{\tau_{\mathrm{R}}} q(x(\tau), t) \frac{\partial x}{\partial \tau} d \tau, \quad M=-k \int_{0}^{\tau_{\mathrm{R}}} x(\tau) q(x(\tau), t) \frac{\partial x}{\partial \tau} d \tau,
$$

where the derivative $\partial x / \partial \tau$ can be obtained from Eq. (9), whereas $\tau_{\mathrm{R}}$ is the time delay at the trailing edge $\mathrm{R}(x=-a)$, which can be also calculated from Eq. (9).

Due to the presence of the time delay the equations (10) are frequently referred to as 'tyre with memory effect'.

\section{LINEAR STABILITY}

\subsection{Car-trailer combination}

By considering the 'memory-effect' in tyre deformation the equations of motion (Eq. (3)) of the car-trailer system is transformed into a system of delay differential equations with a distributed time delay. Afterwards, we linearise the equations of motion around the rectilinear motion (i.e. the trivial solution), which provides us the following matrix differential equation:

$$
\mathbf{M} \ddot{\mathbf{y}}(t)+\mathbf{S y}(t)=\mathbf{Q}\left(\mathbf{y}_{\tau}\right),
$$

where $\mathbf{y}(t)$ is the vector of the remaining state variables of the linearised system:

$$
\mathbf{y}(t)=\left(\begin{array}{lll}
Y_{1}(t) & \psi_{1}(t) & \psi_{2}(t)
\end{array}\right)^{T} .
$$

The mass matrix $\mathbf{M}$ can be expressed as

$$
\mathbf{M}=\left(\begin{array}{ccc}
m_{1}+m_{2} & -m_{2} h & -m_{2} l_{\mathrm{c}} \\
-m_{2} h & J_{\mathrm{C}_{1}}+m_{2} h^{2} & m_{2} h l_{\mathrm{c}} \\
-m_{2} l_{\mathrm{c}} & m_{2} h l_{\mathrm{c}} & J_{\mathrm{C}_{2}}+m_{2} l_{\mathrm{c}}{ }^{2}
\end{array}\right) .
$$

whereas the stiffness matrix $\mathbf{S}$ reads

$$
\mathbf{S}=2 a k\left(\begin{array}{ccc}
3 & f-b-h & -l \\
f-b-h & f^{2}+b^{2}+h^{2}+\frac{2}{3} a^{2} & l h \\
-l & l h & l^{2}+\frac{1}{3} a^{2}
\end{array}\right) .
$$

The retarded functional $\mathbf{Q}(t-\tau)$ on the right hand side of Eq. (11) can be composed as:

$$
\mathbf{Q}\left(\mathbf{y}_{\tau}\right)=k V \int_{0}^{\frac{2 a}{V}}\left(\mathbf{B}_{0}-V \tau \mathbf{B}_{1}\right) \mathbf{y}_{\tau} \mathrm{d} \tau,
$$

where $\mathbf{y}_{\tau}$ is the solution segment $\mathbf{y}(t-s)$ for $s \in[0, \tau]$ and the coefficient matrices are:

$$
\mathbf{B}_{0}=\left(\begin{array}{ccc}
3 & f-b-h+2 a & -l+a \\
f-b-h+2 a & f^{2}+b^{2}-h^{2}+2 a(f-b+a) & h(l-a) \\
-l+a & h(l-a) & (l-a)^{2}
\end{array}\right)
$$

and

$$
\mathbf{B}_{1}=\left(\begin{array}{ccc}
0 & 0 & 0 \\
2 & a-b+2 a & 0 \\
1 & -h & -l+a
\end{array}\right) .
$$

Then, by taking an exponential trial solution, we can compose the characteristic function which is used to determine the stability of the rectilinear motion for different values of the longitudinal velocity $V$ and the payload position parameter $p$, which is introduced as $p=l / l_{\mathrm{C}}$ i.e. $p<1$ if the centre of gravity of the trailer is before the axle of the wheels and $p>1$, if the centre of gravity is located behind the axle of the wheels.

Stability charts were constructed by finding the zeros of the characteristic function in the $(V, p)$ parameter plane using the multidimensional bisection method (Bachrathy and Stépán, 2012). It can be shown that at large longitudinal velocities two unstable parameter domains occur, out of which one corresponds to a saddle-node (SN) bifurcation, whereas the other belongs to Hopf bifurcation. It is worth to note that the detection of these two domains is also possible via simpler tyre models which do not include the memory effect of the tyres. Nevertheless, new unstable parameter domains occur at small velocities, which can be only captured by taking into account the 
(a)

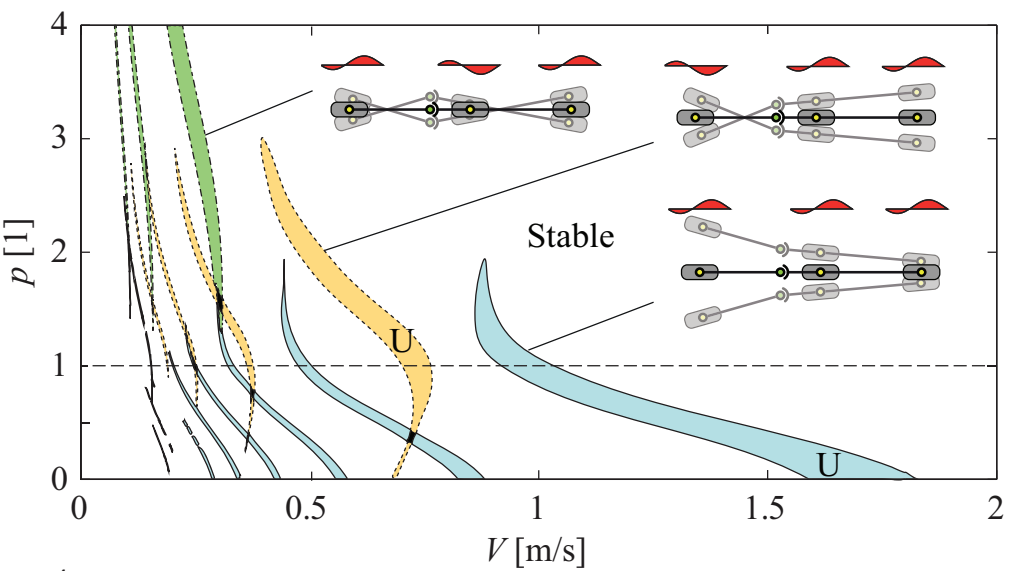

(b)

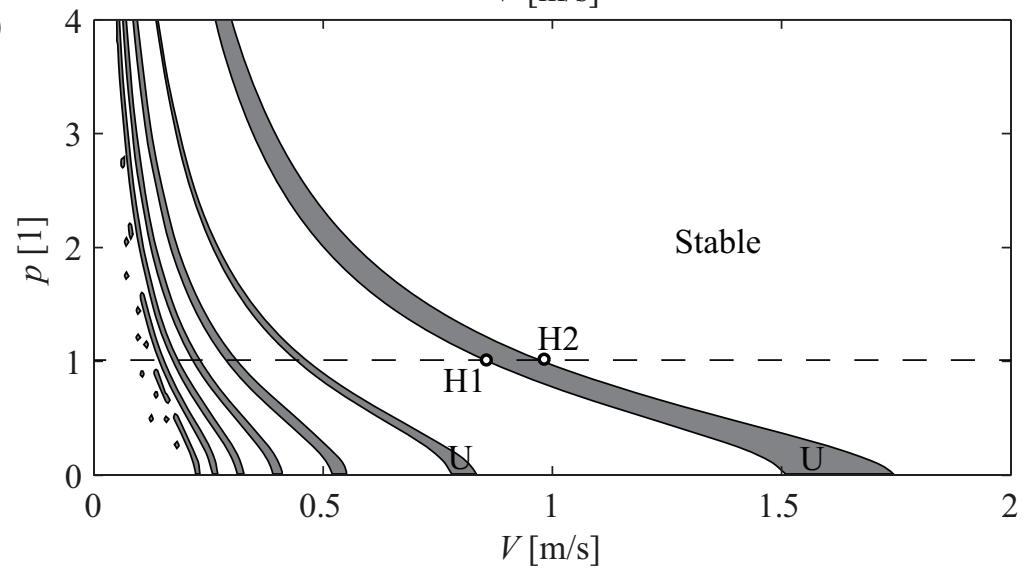

Figure 4. Stability charts of the rectilinear motion of the car-trailer combination (a) and the towed trailer (b), respectively for parameters $m_{1}=1600[\mathrm{~kg}], J_{\mathrm{C}_{1}}=24576\left[\mathrm{kgm}^{2}\right], m_{2}=m=400[\mathrm{~kg}], J_{\mathrm{C}_{2}}=J_{\mathrm{C}}=$ $800\left[\mathrm{kgm}^{2}\right], f=1.4[\mathrm{~m}], b=1.6[\mathrm{~m}], h=1.8[\mathrm{~m}], l=2[\mathrm{~m}], a=0.05[\mathrm{~m}], k=2 \cdot 10^{7}[\mathrm{~N} / \mathrm{m}]$. For the car-trailer combination the different colors correspond to different 'critical' (i.e. unstable) mode shapes, which are depicted with the characteristic tyre deformations.

regenerative effect in the tyre-ground contact patch. These unstable parameter domains, all related to Hopf bifurcations, are shown in detail in Figure 4 where we also represent the corresponding unstable vibration modes based on numerical simulations. As it is common for time-delayed systems, it can be shown that if the longitudinal velocity tends to zero $(V \rightarrow 0)$ an infinite number of such unstable domains emerge.

\subsection{Towed trailer}

The same methods are deployed to investigate the stability of the towed trailer. The linearised equation of motion can be expressed as

$$
\left(J_{\mathrm{C}}+m l_{\mathrm{C}}^{2}\right) \ddot{\psi}(t)=k \int_{0}^{2 a / V}(a-l-V \tau)(V \tau \psi(t)-(a-l)(\psi(t)-\psi(t-\tau))) V d \tau .(18
$$

Again, we use the characteristic function to determine the stability boundaries. As in the case of the car-trailer system, the stability boundaries are determined in terms of the longitudinal velocity $V$ and the payload position parameter $p$. For this case, it can be proven that for real vehicle parameters (i.e positive tyre stiffness $k$, contact patch half-length $a$ and caster length $l$ ) no saddle-node bifurcations occur. However, we still can capture unstable parameter domains at low velocities that all relate to Hopf bifurcations causing the oscillation of the trailer. In can be seen, that the structure of the stable and unstable parameter domains is similar to the car-trailer system, i.e. as the velocity tends to zero $(V \rightarrow 0)$ an infinite number of unstable domains emerge. These results were also verified by former studies focusing on the analysis of wheel-shimmy with a time-delayed tyre model (Takács, 2010). 


\section{NONLINEAR ANALYSIS}

\subsection{The discretisation method}

In order to get further insight into the dynamics of tyre-excited vibrations we also investigated the nonlinear dynamics. To simplify our calculations from now on we only concentrate on the analysis of the towed trailer. Our aim is to capture the limit cycles emerging at the Hopf bifurcations. A possible approach to do this is a centre-manifold reduction of the time-delayed system, and composing the corresponding normal form, by which it is possible to establish the behaviour of the system near to the bifurcation points, i.e. whether the emerging limit cycles are sub- or supercritical (Hale and Lunel, 1993), (Campbell, 2009). Alternatively, the method of multiple scales can be applied, which would provide us essentially the same results without the calculation of the centre manifold itself (Das and Chatterjee, 2002). These methods can be very powerful in those cases when the results can be obtained analytically as they give more insight to the dynamics of the system than using numerical methods. However, in our case the analytical solution becomes intractable due to the distributed delay, which makes it necessary to use numerical methods for the evaluation; therefore, this method loses from its efficiency.

Instead, in our study we focused on the continuation of the limit cycles itself by using the method of collocation on the equation of motion given in Eq. (18) with the travelling wave solution Eq. (8). An alternative solution would be to use the PDE (6) instead of the travelling wave solution and discretise the contact patch which would enable us to use simpler mathematical formulations. However it would be very disadvantageous due to the large dimension of the discretised system and high computation time (Beregi, 2014).

The usage of the travelling wave solution given by can be practically seen as a re-parametrisation of the contact patch by using the formula in Eq. (9) to describe the relationship between the spatial coordinate $x$ and the time delay $\tau$. Taking the advantage of this, we can still use coordinate $x$ as our integration variable and the fixed domain of $x \in[-a, a]$ instead of the state-dependent limit in Eq. (10). Thus, we use the following form of the governing equations:

$$
\dot{\Omega}(t)=\frac{k}{J_{\mathrm{C}}+m l_{\mathrm{C}}^{2}} \int_{-a}^{a}(x(\tau)-l) q(x(\tau), t) d x, \quad \dot{\psi}(t)=\Omega(t) .
$$

In this case the solution is approximated over the period by Fourier-series which enabled us to use an equidistant grid in the time-domain. Thus, after introducing the dimensionless time variable $\tilde{\vartheta}=2 \pi t / T$ the period $\tilde{\vartheta} \in[0,2 \pi]$ is divided into $N$ intervals with $N+1$ grid points as $0=\tilde{\vartheta}_{0}<\tilde{\vartheta}_{1}<\ldots<\tilde{\vartheta}_{N-1}<\tilde{\vartheta}_{N}=2 \pi$.

Using the function values of the angular velocity $\Omega(t)$ and the deflection angle $\psi(t)$ at the grid points $\tilde{\vartheta}_{i}$ we can construct the vectors $\Omega$ and $\psi$ as $\Omega=\left(\Omega_{0}, \Omega_{1}, \ldots \Omega_{i} \ldots \Omega_{N}\right)^{\mathrm{T}}$ and $\boldsymbol{\psi}=$ $\left(\psi_{0}, \psi_{1}, \ldots \psi_{i} \ldots \psi_{N}\right)^{\mathrm{T}}$, where $\Omega\left(\tilde{\vartheta}_{i}\right)=\Omega_{i}$ and $\psi\left(\tilde{\vartheta}_{i}\right)=\psi_{i}$ are the angular velocity and the deflection angle at the $i^{\text {th }}$ time point $\tilde{\vartheta}_{i}$ for $i=1, \ldots, N$.

Taking this into account, the time-derivatives are calculated with the help of the first order Fourier differentiation matrix $\mathbf{D}^{\mathrm{F} 1}$ (Trefethen, 2000) as $\dot{\boldsymbol{\Omega}} \cong 2 \pi / T \mathbf{D}^{\mathrm{F} 1} \Omega$ and $\dot{\psi} \cong 2 \pi / T \mathbf{D}^{\mathrm{F} 1} \boldsymbol{\psi}$.

The time-delayed variables $\Omega(t-\tau)$ and $\psi(t-\tau)$ are obtained by wrapping around the time over the discretised period. Firstly, we normalize the time delay by the period $T$ as $\tau_{n}=\tau / T$. Then, the time-delayed values are obtained as $\Omega_{\tau i}=\Omega\left(\tilde{\vartheta}_{i}-\tau_{n} 2 \pi\right)=\Omega_{i-\tau_{n} N}$ and $\psi_{\tau i}=$ $\psi\left(\tilde{\vartheta}_{i}-\tau_{n} 2 \pi\right)=\psi_{i-\tau_{n} N}$. It is assumed here, that the values of $\tau_{n}$ are integers, i.e. the timedelayed variables are also evaluated at the representation points. However, this condition would not generally apply for the state-dependent time delay $\tau_{R}$ at the trailing edge $(x=-a)$, which makes it necessary to estimate the angular velocity $\Omega_{\tau_{n *}}$ and the deflection angle $\psi_{\tau_{n *}}$ by linear interpolation between the closest bounding collocation points. Then, the integral in Eq. (19) can be calculated by numerical methods, such as the trapezoid formulae which is applied in our study. Alternatively, one can use formulae from the Fourier scheme for integration and interpolation; however, these are more difficult to implement.

Considering these, the discretised system of the governing equations can be expanded as

$$
\frac{2 \pi}{T} \sum_{j=1}^{N} D_{i j}^{\mathrm{F} 1} \Omega_{j}=\frac{k}{J_{\mathrm{C}}+m l_{\mathrm{C}}^{2}} \sum_{k=1}^{\tau_{n *}} w_{k}\left(x_{k}-l\right) q_{k}, \quad \frac{2 \pi}{T} \sum_{j=1}^{N} D_{i j}^{\mathrm{F} 1} \psi_{j}=\Omega_{j},
$$



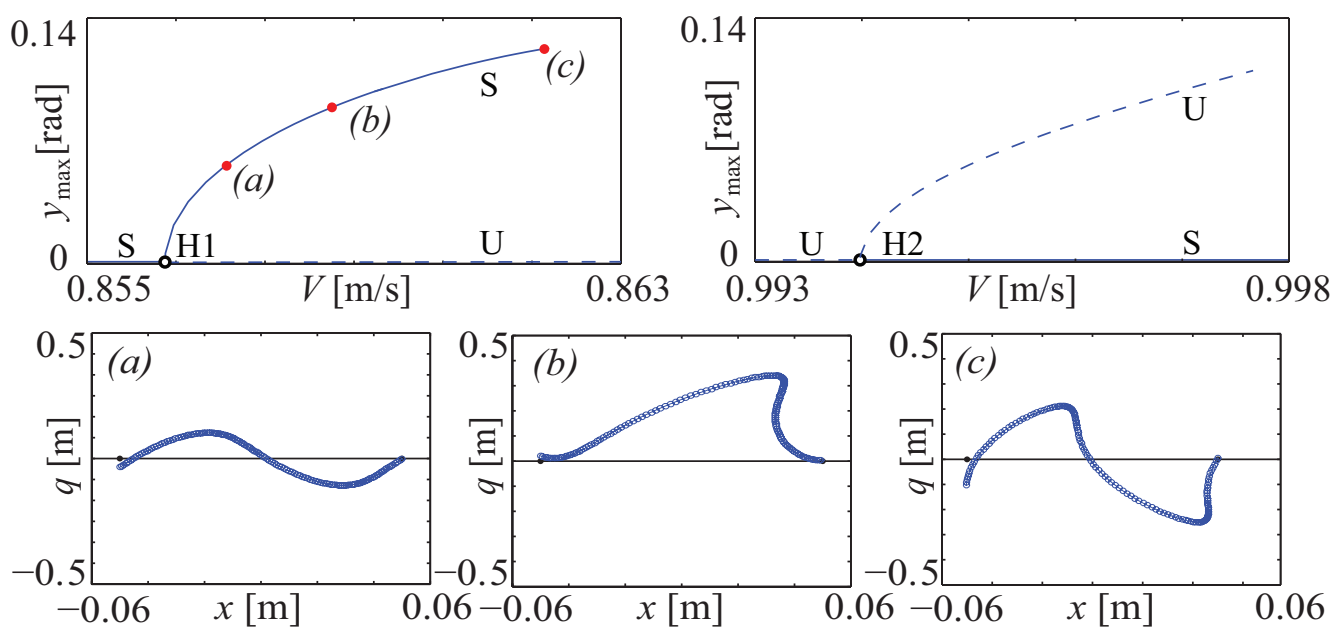

Figure 5. The branches of limit cycles emerging from the Hopf points $\mathrm{H} 1$ and $\mathrm{H} 2$ and the corresponding tyre deformations. The stable solutions are marked with continuous line and S, whereas the dashed lines and the notation $\mathrm{U}$ correspond to unstable solutions.

with the additional phase condition of $\Omega_{N}=0$, where where the values $x_{k}$ and $q_{k}$ are composed according to the time-delayed formulae Eq. (8) and Eq. (9):

$$
\begin{gathered}
q_{k}=V \tau_{k} \sin \psi_{k}-(a-l) \sin \left(\psi_{k}-\psi_{\tau k}\right), \\
x_{k}=l-V \tau \cos \psi_{k}+(a-l) \cos \left(\psi_{k}-\psi_{\tau k}\right),
\end{gathered}
$$

whereas $w_{k}$ are the weight factors of the numerical integration method. It is worth to note that in some cases a more general phase condition (e.g. an integral phase condition) might be needed. The periodicity of the solution is already assumed by using Fourier-series; therefore, no additional condition is required.

\subsection{Results of the continuation}

Keeping the payload position parameter constant at $p=1$ we determined the stable and unstable domains with respect to the longitudinal velocity $V$. By means of our analysis a set of limitcycle pairs could be located at each unstable parameter range. Out of each pair of limit cycles one is supercritical (stable) and the other is subcritical (unstable). Figure 5 shows the branches of limit cycles emerging from the bifurcation points $\mathrm{H} 1$ and $\mathrm{H} 2$, which belong to the stability boundary that corresponds to the largest towing speed $V$ (see Fig. 4 for reference). By plotting the tyre deformation over the identified periodic orbits it was found, that at smaller amplitudes the deformation has a sinusoid-like shape (Fig. 5 (a)) which result agrees with the ones provided by studying the eigensystem of the linearised system.

However we can notice that at larger amplitudes the tyre deformation 'turns back' (see Fig. 5 (b)), i.e. there are certain ranges where two contact points correspond to a given value of of $x$. A particular advantage of using the travelling wave solution over the PDE that these cases can be handled too, as long as the time-delay $\tau_{R}$ at the trailing edge $\mathrm{R}$ can be calculated straightforwardly. This can be ensured for a relatively large range of amplitudes, however, there is an upper limit for the usability of our model, where the deformation starts to 'turn back' at the trailing edge as well (see Fig. 5 (c)).

Nevertheless, this 'extreme' case of tyre deformation can be regarded as unrealistic in terms of our physical experience. It can be also noticed, that the amplitude of the vibrations very rapidly reaches a level that would involve significant side-slip of the tyre in real-life systems. This leads us to the conclusion, that although they are mathematically 'correct' solutions, the range of physical validity of the captured periodic orbits are relatively small, and further analysis is needed to achieve a better correspondence to the real-life behaviour. 


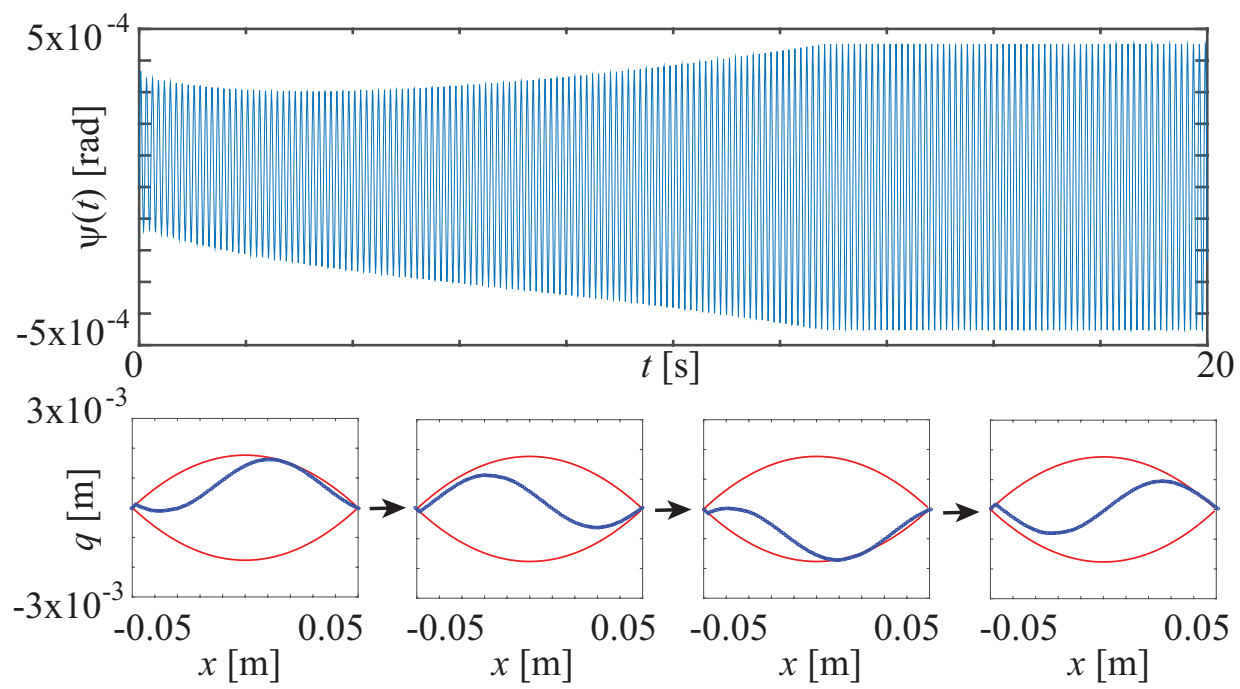

Figure 6. The simulation results with the enhanced brush tyre model for $V=0.8568[\mathrm{~m} / \mathrm{s}]$ with a friction coefficient of $\mu=0.6$, initial conditions: $\Omega(0)=0.02[\mathrm{rad} / \mathrm{s}], \psi(0)=0$.

\subsection{Numerical simulation with side-slip considered}

To take into account the partial slip of the tyres, the brush tyre model is modified by setting a boundary for the tyre deformation, as the local forces from the deformation are limited by the Coulomb-friction between the tyre and road surface. For pure side-slip, we assume parabolic lateral deformation in the contact patch, which can be well matched with experimental results (Pacejka, 2002). We use these parabolae (see the red lines in Fig. 6) as limits for the actual tyre deformation by which we imply that the deformation is zero not only at the leading edge $\mathrm{L}$ $(x=a)$, but at the trailing edge $\mathrm{R}(x=-a)$ too, whereas it is also assumed that the friction coefficient is the same for sticking and slipping (Beregi, 2014).

In Figure 6 we present the results of the numerical simulation of a trailer towed with a constant speed $V=0.8568[\mathrm{~m} / \mathrm{s}]$, which corresponds to case $(a)$ presented in Figure 5. It was found, that by using parameters from the linearly unstable domains the solution converges to a stable periodic orbit. It can be observed, that within a period one or multiple ranges of the contact patch slip, which limits the tyre deformation at the level according to the maximum locally achievable friction-force. The amplitude of the vibrations are also considerably smaller than in the case when pure rolling was assumed due to the fact, that the side-slip consumes the energy of the mechanical system. Moreover, we can say, that with the studied parameters the oscillation is restricted to such a small level (i.e. $\|\psi(t)\|<5 \times 10^{-4}$ ) that even if we neglect tyre damping it is very unlikely to cause stability-problems in real-life systems.

\section{CONCLUSIONS}

In our study we showed how the so-called 'memory effect' in the tyre-ground contact patch leads to self excited oscillations of the vehicles. By implementing the brush tyre model new unstable parameter domains were found for the car-trailer system at low velocities, which can be categorized into three types according to the shape of the unstable vibration mode.

Additionally, we studied the nonlinear dynamics of a single towed trailer as well. We were able to show that the simpler mechanical model still contains unstable parameter domains caused by self-excited vibrations of the tyre and the stability boundaries correspond to Hopf bifurcations. The emerging limit cycles were captured by applying the method of collocation on the governing equations. We could notice that the limit cycles rapidly reach an amplitude which belongs to large tyre deformations that would involve significant side-slip of the tyre in real-life systems. Consequently, the range of validity of the solutions, where the condition of pure rolling holds, is limited.

The results were compared with numerical simulation using the enhanced the brush tyre model taking into consideration the possible side-slip of tyre particles as well. It could be observed that 
by choosing parameters from linearly unstable domains after perturbation the solution converges to a stable limit cycle. However, the amplitude of the vibrations are considerably smaller than in the case when pure rolling is assumed. Hence, we can conclude that with the studied parameters the oscillation is restricted to such a small level that even if we neglect tyre damping it is very unlikely to cause stability-problems in real-life systems. In spite of this, these vibrations may be still relevant due to the unwanted noise-generation or the increased tyre wear and fuel consumption.

The dynamics of the towed trailer with a stick-slip tyre model could be subject of further analysis by calculating the branch of the limit-cycles predicted by numerical simulation. However, since we are dealing with a piecewise-smooth system (Leine, 2006) one may find it demanding to handle it as a boundary value problem as the time-delayed formulae can be used only for the sticking parts of the contact patch. Therefore, we cannot use the original travelling wave solution for those tyre particles which stick back to the ground after they have been slipping. Instead, a modified delayed formula should be composed, based on the former state, when the particles were sticking back to the ground. Although this approach leads to very complicated equations, it may be still rewarding to implement, as by doing so we could benefit from the advantages of having a system with reduced dimension, which may enables us to obtain more accurate results.

Acknowledgement This research was partly supported by the János Bolyai Research Scholarship of the Hungarian Academy of Sciences and by the Hungarian National Science Foundation under grant no. OTKA PD105442.

The publication of the work reported herein has been supported by ETDB at BME.

\section{REFERENCES}

Bachrathy, D. \& G. Stépán (2012). Bisection method in higher dimensions and the efficiency number. Periodica Polytechnica, 81-86.

Beregi, S. (2014). The impact of the dynamics of rubber tyres on the stability of vehicles. Master's thesis, Budapest University of Technology and Economics, Hungary.

Campbell, S. (2009). Calculating Centre Manifolds for Delay Differential Equations Using Maple, Chapter 8 in in Delay Differential Equations, Recent Advances and New Directions. Springer-Verlag, New York.

Coetzee, E., B. Krauskopf, \& M. Lowenberg (2006). Nonlinear analysis of a coupled nose landing gear-fuselage system. Proceedings of ICGF.

Das, S. \& A. Chatterjee (2002). Multiple scales without center manifold reductions for delay differential equations near hopf bifurcations. Nonlinear Dynamics 30: 323-335, Kluwer Academic Publishers.

Gantmacher, F. (1975). Lectures in analytical mechanics. MIR Publishers, Moscow.

Hale, J. \& S. Lunel (1993). Introduction to Functional Differential Equations. Springer-Verlag, New York.

Howcroft, C., B. Krauskopf, M. Lowenberg, \& S. Neild (2012). Effects of freeplay on aircraft main landing gear stability. Proceedings of AIAA 2012, AIAA, Minneapolis, Minnesota, USA (AIAA 2012-4730.), 1-16.

Leine, R. I. (2006). Bifurcatons of equilibria in non-smooth continuous systems. Physica D, $121-137$.

Pacejka, H. B. (2002). Tyre and Vehicle Dynamics. Elsevier Butterworth-Heinemann, Oxford.

Rossa, F. D., G. Mastinu, \& C. Piccardi (2012). Bifurcation analysis of an automobile model negiotating a curve. Vehicle System Dynamics: International Journal of Vehicle Mechanics and Mobility.

Takács, D. (2010). Dynamics of towed wheels - Nonlinear theory and experiments. Ph. D. thesis, Budapest University of Technology and Economics, Hungary.

Takács, D. \& G. Stépán (2012). Contact patch memory of tyres leading to lateral vibrations of four-wheeled vehicles. Philosophical Transactions of the Royal Society A: Mathematical, Physical and Engineering Sciences.

Terkovics, N., S. Neild, M. Lowenberg, \& B. Krauskopf (2012). Bifurcation analysis of a coupled nose landing gear-fuselage system. Proceedings of AIAA, Minneapolis, Minnesota, USA, 1-14.

Trefethen, L. (2000). Spectral Methods in Matlab. Society for Industrial and Applied Mathematics, Philadelphia. 